N.EINNAMI AND K. SHIGA

KODAI MATH. J.

8 (1985), 259-263

\title{
THE AXIOM OF GENERALIZED HYPERSPHERES IN RIEMANNIAN GEOMETRY
}

\author{
By Nobuhiro InNAMi AND KoIchi Shiga
}

\section{Introduction.}

A characterization of spaces of constant curvature is the most classical and interesting subject in Riemannian geometry ([1], [2], [3], [9], [10], [11], [12], [13], [14], [15], etc.). Many axioms which characterize spaces of constant curvature have been found out. The axiom of $n$-planes by Cartan [3] and the axiom of $n$-spheres by Leung-Nomizu [11] are well-known. In the present note we shall establish the axiom of generalized hyperspheres and apply it to geodesic spheres and horospheres to obtain characterizations of spaces of constant curvature.

We prepare the notation for giving our axiom. Let $M$ be a Riemannian manifold of dimension $m \geqq 3$ and $N$ be a hypersurface (at least of class $C^{2}$ ) in $M$. Suppose that $N$ has a unit normal vector field $v$. For a characterization of spaces of constant curvature we may assume without loss of generality that $\phi: N \times[0, \varepsilon) \rightarrow M$ given by $\phi(q, t):=\exp t v_{q}$ is diffeomorphic onto its image for some positive $\varepsilon$, because curvature properties are local ones. If $\phi_{t}(q):=\phi(q, t)$ for any $q \in N$ and for each $t$, then $N_{t}:=\phi_{t}(N)$ is a hypersurface in $M$ for each $t$. The family $\left\{N_{t} ; t \in[0, \varepsilon)\right\}$ will be called the family of generalized hypersurfaces associated with $\phi$. Set $c_{q}(t):=\exp t v_{q}$ for each $q \in N$.

We now introduce the axiom.

Axiom of generalized hyperspheres. For every point $p \in M$ and every $(m-1)$ dimensional subspace $T_{p}^{\prime}$ of $T_{p} M$, there exists a hypersurface $N$ through $p$ such that $T_{p} N=T_{p}^{\prime}$ and $N_{t}$ is umbilical at $c_{p}(t)$ for each $t \in[0, \varepsilon)$.

In this axiom there are many choices of $N$, since the axiom does not require that $N_{t}$ is umbilical at $c_{q}(t)$ for any point $q \neq p$ in $N$.

Then we shall prove

THEOREM 1. Let $M$ be a Riemannian manifold of dimension $m \geqq 3$. If $M$ satısfies the axiom of generalized hyperspheres, then $M$ is a space of constant curvature.

Applying Theorem 1 to geodesic spheres, we shall obtain

CoROllary 2. Let $M$ be a Riemannian manifold of dimension $m \geqq 3$. If all

Received July 20, 1984 
small geodesic spheres in $M$ are totally umbilical, then $M$ is a space of constant curvature.

Tachibana-Kashiwada [15] have proved this corollary in the case where $M$ is an Einstein manifold.

COROllary 3. Let $M$ be a Riemannian manifold of dimension $m \geqq 3$. If all small concentric geodesic spheres in $M$ are conformal along any geodesic emanating from the common center, then $M$ is a space of constant curvature.

We shall also have the analogous results for horospheres instead of geodesic spheres. Let $M$ be a simply connected complete Riemannian manifold without conjugate points. Then $M$ is diffeomorphic to $\boldsymbol{R}^{m}$ where $m:=\operatorname{dim} M$, and all geodesics are minimizing. Let $\gamma:(-\infty, \infty) \rightarrow M$ be a unit speed geodesic. The Busemann function $f_{\gamma}$ on $M$ is given by $f_{\gamma}():.=\lim \{d(., \gamma(t))-t\}$. Set $H_{s}:=$ $f_{\gamma}^{-1}(s)$ for each $s \in \boldsymbol{R}$, which is called the horosphere through $\gamma(s)$ with central ray $\gamma . f_{\gamma}$ is in general known to be at least of class $C^{1}$. However, under the additional condition on $M$ ((for example, nonpositive curvature), $f_{r}$ is of class $C^{2}([5],[8])$. We say that a unit speed geodesic $\alpha:(-\infty, \infty) \rightarrow M$ is an asymptote to $\gamma$ if $\dot{\alpha}(t)=-\operatorname{grad} f_{\gamma}(\dot{\alpha}(t))$ for each $t \in \boldsymbol{R}$. There passes a unique asymptote through given point to $\gamma$ in $M$.

COROLLARY 4. Let $M$ be a simply connected complete Riemannian manifold without conjugate points and of dimension $m \geqq 3$. If all horospheres in $M$ are of class $C^{2}$ and totally umbilical, then $M$ is a space of constant curvature.

COROLlARY 5. Let $M$ be a simply connected complete Riemannian manifold without conjugate points and of dimension $m \geqq 3$. If all horospheres are of class $C^{2}$ and if all concentric horospheres in $M$ are conformal along any asymptote to the common central ray, then $M$ is a space of constant curvature.

Of course, our assumption makes no sense if $\operatorname{dim} M=2$. In that case Green [7] states a characterization of surfaces of negative constant curvature under the additional conditions on horosperes.

Corollary 2 and 4 are direct consequences of Theorem 1 . And, under the assumption of Corollary 3 and 5 , it will turn out that geodesic spheres and horospheres are totally umbilical (see Lemma 6).

\section{Preliminalies.}

Our methods of the study will be based on a paper of Eschenburg-O'Sullivan [6]. The following arguments can be seen in [6]. Let $M$ be a Riemannian manifold and let $N$ be a hypersurface (at least of class $C^{2}$ ) in $M$. Suppose that $N$ has a unit normal vector field $v$. Let $\phi: N \times[0, \varepsilon) \rightarrow M$ be a map given by $\phi(q, t):=\exp t v_{q}$ for any $(q, t) \in N \times[0, \varepsilon)$. For each $t$ let $\phi_{t}: N \rightarrow M$ be a map 
given by $\phi_{t}(q):=\phi(q, t)$ for any $q \in N$. Suppose that $N_{t}:=\phi_{t}(N)$ is a hypersurface in $M$ for each $t$. Define a unit vector field $V$ on $\phi(N \times[0, \varepsilon))$ by setting $V(\phi(q, t)):=\dot{c}_{q}(t)$, where $c_{q}:[0, \varepsilon) \rightarrow M$ is the geodesic with $c_{q}(t):=\exp t v_{q}$. Obviously, $V \mid N_{t}$ is a unit normal vector field on $N_{t}$ for each $t$. Fix a point $p \in N$ and let $c:=c_{p}$. For each $t$, if $D(t):=\phi_{t^{*}} \cdot P_{t}^{-1}$ where $P_{t}$ is the parallel translation along $c$ from $p$ to $c(t)$, then $D(t)$ is an isomorphism of $T_{c(t)} N_{t}$ onto itself. For a vector $x \in T_{p} N$, if $X(t):=P_{t} x$ for any $t$, then $D X(t)=\phi_{t^{*}}(x)$ is a Jacobi vector field along $c$. Hence

$$
D^{\prime \prime} X+R(D X, \dot{c}) c=0
$$

Thus, if $Y:=D X$,

$$
\left(D^{\prime \prime} D^{-1}\right) Y+R(Y, \dot{c}) c=0 .
$$

Also, if $A(t)$ is the second fundamental form of $N_{t}$ relative to $V \mid N_{t}$ for each $t$,

$$
D^{\prime} X(t)=\nabla_{\dot{c}(t)} D X(t)=\nabla_{D X(t)} V(\phi(p, t))=-A(t)(D X(t)) .
$$

Hence, if $U(t):=D^{\prime} D^{-1}(t)$, we have

$$
U(t)=-A(t) .
$$

If we differentiate $U$ covariantly and substitute into (*),

$$
U^{\prime} Y \dot{+} U^{2} Y+R(Y, \dot{c}) c=0 .
$$

Therefore, if $R_{c(t)}$ is a linear map of $T_{c(t)} N_{t}$ into itself given by $R_{c(t)}(y):=$ $R(y, \dot{c}) \dot{c}$ for any vector $y \in T_{c(t)} N_{t}$, then

$$
U^{\prime}+U^{2}+R_{c}=0
$$

\section{Proofs and Lemma.}

In this section we shall give the proof of Theorem 1 and Lemma 6. Corollary 2 and 4 are direct applications of Theorem 1, so we may omit the proofs of them. Corollary 3 and 5 will be also reduced to Corollary 2 and 4 by Lemma 6 , so it suffices to prove Lemma 6 .

\section{Proof of Theorem 1.}

From Schur's theorem (see [4], p. 16) it is sufficient to prove that the sectional curvature depends only on the point $p \in M$. Let $x \in T_{p} M$ be an arbitrary unit vector and let $T_{p}^{\prime}$ be the subspace of $T_{p} M$ orthogonal to $x$. Take a hypersurface $N$ and a variation $\phi$ along $N$ as in the axiom of generalized hyperspheres. Set $c(t):=\phi(p, t)=\exp t v_{p}$. Since the submanifold $N_{t}$ is umbilical at $c(t), U(t)=$ $\lambda(t) I$ for some function $\lambda$ along $c\left(\right.$ see $\left.\left(2^{*}\right)\right)$. From $\left(3^{*}\right), R_{c}=\left(-\lambda^{\prime}-\lambda^{2}\right) I$. Hence, if $n:=m-1$, then

$$
\operatorname{Ric}(\dot{c}, \dot{c})=n\left(-\lambda^{\prime}-\lambda^{2}\right) .
$$

Thus we have 


$$
R_{c}=(1 / n) \operatorname{Ric}(\dot{c}, \dot{c}) I .
$$

In particular,

$$
R(y, x) x=(1 / n) \operatorname{Ric}(x, x) y
$$

for any vector $y \in T_{p}^{\prime}$. If $y \in T_{p} M$ is a unit vector orthogonal to $x$,

$$
\operatorname{Ric}(x, x)=n\langle R(y, x) x, y\rangle=n\langle R(x, y) y, x\rangle=\operatorname{Ric}(y, y) .
$$

This implies that the Ricci curvature at $p$ is independent of the direction. Again, by the identity $\left(4^{*}\right)$, the sectional curvature depends sonly on the point of $M$. This completes the proof.

Now we prove the following.

LEMMA 6. Let $N$ be a hypersurface in a Riemannian manifold $M$ and let $\phi$ be a normal geodesic variation along $N$. If $\phi_{t}: N \rightarrow N_{t}$ is conformal for each $t$, then $N_{t}$ is totally umbilical for each $t$.

Proof. Let $p \in N$ and $c(t):=\exp t v_{p}$ for any $t$. Let $D(t):=\phi_{t^{*}} \cdot P_{t}^{-1}$ as in Section 1. The assumption implies that there exists a positive function $\rho$ on $N \times[0, \varepsilon)$ such that $\left\langle\phi_{t^{*}}(x), \phi_{t^{*}}(y)\right\rangle=\rho(q, t)\langle x, y\rangle$ for any $q \in N$ and for any vectors $x$ and $y \in T_{q} N$. If $X(t):=P_{t} x$ and $Y(t):=P_{t} y$ for each $t$ and vectors $x$ and $y \in T_{p} N$, then

$$
\langle D X(t), D Y(t)\rangle=\rho(p, t)\langle x, y\rangle .
$$

If we differentiate both side with respect to $t$,

$$
\left\langle D^{\prime} X(t), D Y(t)\right\rangle+\left\langle D X(t), D^{\prime} Y(t)\right\rangle=\rho^{\prime}(p, t)\langle x, y\rangle
$$

for each $t$. Therefore, since $U:=D^{\prime} D^{-1}$ is symmetric,

$$
2\langle U D X(t), D Y(t)\rangle=\rho^{\prime}(p, t)\langle x, y\rangle=\left(\rho^{\prime}(p, t) / \rho(p, t)\right)\langle D X(t), D Y(t)\rangle
$$

for each $t$. Since $D(t)$ is invertible for each $t$,

$$
U(t) z=\left(\rho^{\prime}(p, t) / 2 \rho(p, t)\right) z
$$

for every vector $z \in T_{c(t)} N_{t}$. Therefore $N_{t}$ is totally umbilical for each $t$, because $U(t)$ is the second fundamental form of $N_{t}$ relative to $-\dot{c}(t)$ (see $\left.\left(2^{*}\right)\right)$.

\section{REFERENCES}

[1] Beltrami, E., Teoria degli spazii di curvatura costante, Annali di Matem., (2) 2 (1868-69), 232-255.

[2] Busemann, H., The geometry of geodesics, Academic Press, New York, 1955.

[3] CARTAN, É., Leçons sur la geometrie des espaces de Riemann, Gauthier-Villars, 1928.

[4] Chen, B-y., Geometry of submanifolds, Marcel Dekker, New York, 1973. 
L5] Eschenburg, J.-H., Horospheres and the stable part of the geodesic flow, Math. Z., 153 (1977), 237-251.

[6] Eschenburg, J.-H. and O'Sullivan, J.J., Jacobi tensors and Ricci curvature, Math. Ann., 252 (1980), 1-26.

[7] GREen, L.W., Remarks on uniformly expanding horocycle parameterizations, J. Differential Geometry, 13 (1978), 263-271.

[8] Heintze, E. AND H.C. ImHOF, On the geometry of horospheres, J. Differential Geometry, 12 (1977), 481-491.

[9] INNAMI, N., The axiom of $n$-planes and convexity in Riemannian manifolds, J. Math. Soc. Japan, 35 (1983), 85-91.

[10] Klein, F., Über die sogenannte nicht-euklidische Geometrie, Math. Ann., 6 (1873), $112-145$.

[11] Leung, D.S. And K. Nomizu, The axiom of spheres in Riemannian geometry, J. Differential Geometry, 5 (1971), 487-489.

[12] Schlaefli, L., Nota alla Memoria del sig. Beltrami, (Sugli spazii di curvatura costante), Annali di Matem., (2) 5 (1871-73).

[13] Schur, F., Über den Zusammenhang der Räume constanten Riemann'schen Krümmungsmasses mit den projectiven Räumen, Math. Ann., 27 (1886), 537-567.

[14] Tachibana, S., The mean curvature for p-plane, J. Differential Geometry, 8 (1973), 47-52.

[15] Tachibana, S. AND T. Kashiwada, On a characterization of spaces of constant holomorphic curvature in terms of geodesic hypersphere, Kyungpook Math. J., 13 (1973), 109-119.

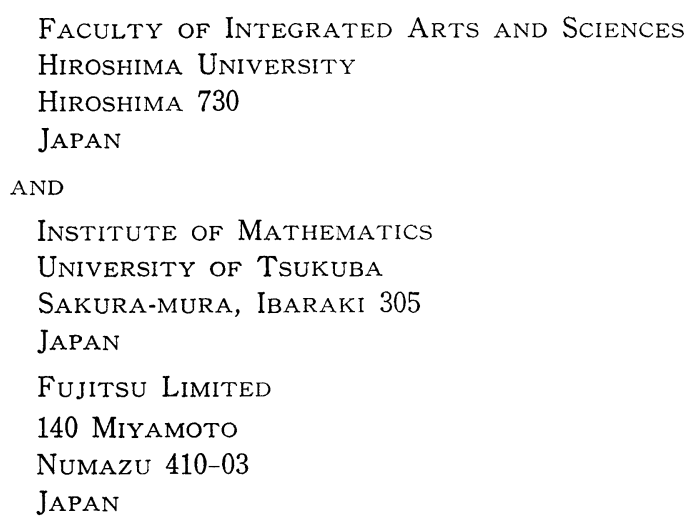

